\title{
Belgeo
}

Revue belge de géographie

$1 \mid 2008$

Aspects of the history of the Belgian geography and cartography

\section{Le Mouvement Géographique, entre géographie et propagande coloniale}

The "Mouvement géographique", between geography and colonial propaganda

\section{Elise Henry}

\section{(2) OpenEdition}

\section{Journals}

Édition électronique

URL : http://journals.openedition.org/belgeo/10172

DOI : $10.4000 /$ belgeo.10172

ISSN : 2294-9135

Éditeur :

National Committee of Geography of Belgium, Société Royale Belge de Géographie

Édition imprimée

Date de publication : 31 mars 2008

Pagination : $27-46$

ISSN : $1377-2368$

Référence électronique

Elise Henry, «Le Mouvement Géographique, entre géographie et propagande coloniale », Belgeo [En ligne], 1 | 2008, mis en ligne le 19 octobre 2013, consulté le 19 avril 2019. URL : http:// journals.openedition.org/belgeo/10172; DOI : 10.4000/belgeo.10172

Ce document a été généré automatiquement le 19 avril 2019

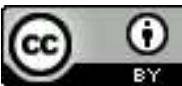

Belgeo est mis à disposition selon les termes de la licence Creative Commons Attribution 4.0 International. 


\title{
Le Mouvement Géographique, entre géographie et propagande coloniale
}

The "Mouvement géographique", between geography and colonial propaganda

\author{
Elise Henry
}

\section{Introduction}

1 Le premier numéro du Mouvement Géographique, sous-titré "Journal populaire des sciences géographiques, illustré de cartes, plans et gravures" paraît à Bruxelles le 6 avril 1884. Ce journal est édité par l'Institut National de Géographie et dirigé par l'un de ses membres, Théodore Falk-Fabian. Le poste de rédacteur en chef est confié à Alphonse-Jules Wauters. En 1890, le Mouvement Géographique est repris par la Compagnie du Congo pour le Commerce et l'Industrie et A.-J. Wauters en devient alors le directeur tout en continuant à remplir sa première fonction.

2 Le Mouvement Géographique paraît tout d'abord un dimanche sur deux, puis à partir de la fin de l'année 1891, il est complété, les autres dimanches, par le Congo illustré "voyages et travaux des Belges dans l'Etat Indépendant du Congo". Ensuite, en 1896, les deux publications fusionnent et le Mouvement Géographique devient une revue hebdomadaire. Sa publication s'étend de 1884 à 1922, avec une interruption lors de la première guerre mondiale. Durant près de 30 ans, A.-J. Wauters fut le principal artisan du Mouvement Géographique qui apparaît ainsi comme l'oeuvre d'un seul homme.

Quels sont les facteurs qui, en cette fin de $19^{\mathrm{e}}$ siècle, poussent à la création et au maintien durant près de 35 ans d'une telle revue de géographie? Qui est Alphonse-Jules Wauters ? Quelle personnalité apparaît en filigrane derrière la grande majorité des articles du Mouvement Géographique? En quoi la nature de son propos en fit-elle une revue si singulière et originale, la distinguant des bulletins des sociétés de géographie de l'époque? Qu'a-t-il apporté, en terme de connaissances, aux géographes et au grand public? Comment l'argument "géographique" a-t-il été utilisé pour convaincre l'opinion 
publique belge de se lancer dans l'aventure coloniale? Et enfin, comment le Mouvement Géographique assura-t-il, sur le plan financier, son maintien durant toutes ces années? Autant de questions qui nous plongent dans l'univers de la période charnière de la fin du $19^{\mathrm{e}}$ siècle et du début du $20^{\mathrm{e}}$ siècle.

Notons que plusieurs publications ont été consacrées au Mouvement Géographique ou à A.-J. Wauters, principalement celles de L. Solvay (1925), R. Cambier (1951), M.-C. Brugaillère (1993) et H. Nicolaï (1993). Chacune d'entre elles aborde certaines de ces questions. Le présent article vient enrichir et compléter l'apport de ces publications en mettant en lumière certains points précis de l'étude approfondie que nous avons menée dans le cadre de notre mémoire, entièrement consacré à l'histoire du Mouvement Géographique ${ }^{1}$. Notre apport particulier porte sur son financement, sur l'identité et les motivations de ses commanditaires ainsi que sur la nature et le contenu de cette revue singulière.

\section{Contexte d'une naissance}

5 La naissance du Mouvement Géographique s'inscrit dans la nouvelle vague d'impérialisme colonial qui, à la fin du $19^{e}$ siècle, secoue l'Europe et touche de nombreux points du globe. C'est le continent africain qui est, cette fois, principalement visé. Conformément aux idées de son temps, Léopold II rêve lui aussi de voir la Belgique acquérir une possession outre-mer. Tout jeune, et avant même d'accéder au trône, il affiche un grand intérêt pour les entreprises coloniales. Ses premiers discours reflètent déjà son désir de voir son pays à la tête d'un domaine colonial qui offrirait non seulement un débouché aux produits de la métropole et des emplois nombreux et lucratifs mais aussi un revenu à la mère patrie. De plus, une colonie favoriserait l'activité commerciale du pays tout en conférant aux capitaux des possibilités de placement avantageuses. Léopold II va donc participer ardemment et par tous les moyens à ce mouvement d'expansion et ce, malgré l'indifférence voire même l'hostilité que manifeste l'opinion publique belge à l'égard de ses visées expansionnistes. Les yeux braqués sur l'exemple de Java ${ }^{2}$ et de ses plantureux bénéfices, il va tout d'abord s'intéresser à l'Extrême-Orient. Mais suite à de multiples tentatives avortées, Léopold II va se tourner vers l'Afrique. Cependant, même lorsqu'il fut à la tête de l'Etat Indépendant du Congo, il ne renonça jamais à l'Extrême-Orient. Léopold II garda sans cesse le regard rivé sur le monde, attentif à toute occasion susceptible de favoriser l'expansion belge, guettant le moment où il pourrait entrer en scène.

6 Vers 1875, Léopold II commence donc à s'intéresser de très près au continent africain. En 1876, près de huit ans avant la création du Mouvement Géographique, le Roi organise en son palais, une conférence de géographie consacrée à l'Afrique. Il y invite principalement des géographes, des explorateurs et des philanthropes connus pour l'intérêt qu'ils portent au continent noir. Il les choisit en Belgique mais aussi en France, en Italie, en Allemagne, en Russie et en Grande-Bretagne. Léopold II y propose la création d'un organisme international, l'Association Internationale Africaine, chargée de coordonner les efforts entrepris en vue "d'explorer scientifiquement les parties inconnues de l'Afrique, de faciliter l'ouverture des voies qui fassent pénétrer la civilisation dans l'intérieur du continent africain et de rechercher des moyens pour la suppression de la traite des nègres en Afrique"3. Cette conférence eut une portée très limitée sur le plan géographique. Cependant, elle marque l'entrée en scène de Léopold II en Afrique. Elle est à l'origine, du moins en partie, de la création de l'Etat Indépendant du Congo, car "la conférence de géographie et ensuite l'Association Internationale Africaine ont donné à Léopold II son 
image, l'image d'un souverain philanthrope, et celle-ci l'aidera puissamment lorsque, en Afrique, il s'occupera de tout autre chose que de philanthropie"4.

7 Au même moment, deux sociétés de géographie sont créées, l'une à Bruxelles et l'autre à Anvers, la première, le 22 août 1876, peu avant la Conférence de Bruxelles ( le 12 septembre), la seconde, peu après, le 2 novembre. Ces créations ne sont pas sans rapport avec l'entreprise coloniale du Roi, que ce dernier plaçait sous le couvert de la géographie. Ces sociétés furent enthousiasmées par le programme humanitaire et scientifique que Léopold II proposa lors de la Conférence. Les géographes belges, par l'intermédiaire de ces sociétés, ont contribué à propager l'idée coloniale et à préparer les Belges, alors réticents à toute entreprise de ce genre, à s'occuper du Congo. Ces sociétés publiaient des bulletins rendant compte des avancées des explorateurs, qu'elles recevaient d'ailleurs avec faste avant leur départ ou pour fêter leur retour. Néanmoins, si les géographes belges ont effectivement contribué à familiariser l'opinion publique avec la politique expansionniste du Roi, Henri Nicolaï tient à préciser qu'il serait abusif de voir, dans la création de ces sociétés de géographie, une action directe de Léopold II, bien qu'il les ait sûrement influencées et utilisées de manière indirecte pour toucher l'opinion publique ${ }^{5}$.

8 L'apparition de sociétés de géographie en Belgique mais aussi un peu partout dans le monde, reflète l'enthousiasme et l'intérêt marqué, en cette fin de $19^{\mathrm{e}}$ siècle, pour les avancées scientifiques. A cette époque, qui fit de l'idée de progrès un leitmotiv, les découvertes géographiques passionnent et suscitent la curiosité. La géographie se détache des récits de voyages pour devenir une véritable science autonome et positive. La création du Mouvement Géographique par l'Institut National de Géographie trouve donc clairement ses origines dans ce contexte de "renouveau géographique". A.-J. Wauters y fait explicitement allusion lorsqu'il expose le programme de la revue lors de la sortie de son premier numéro. Il y place le Mouvement Géographique sous le couvert de la science géographique, qu'il désire montrer sous un jour nouveau, la rendant à la fois attrayante et accessible au grand public.

Mais si la création du Mouvement Géographique reflète cette soif de connaissances et ce "mouvement de renaissance géographique" qui anime la fin du $19^{\mathrm{e}}$ siècle, Wauters vise néanmoins plus loin. En effet, le déferlement d'informations "géographiques" apparaît surtout comme une sorte de "décor" pour ce qui deviendra, en fin de compte, la principale raison d'être du Mouvement Géographique: pousser les Belges à se lancer dans l'aventure coloniale.

10 A cette époque, les visées expansionnistes du Roi ne remportent que très peu de succès auprès de l'opinion publique belge, se heurtant même, parfois, à une ferme opposition. Ce manque d'intérêt pour les entreprises lointaines se retrouve tout autant dans les milieux d'affaires. L'étude de la répartition des capitaux belges à l'étranger à la fin du $19^{e}$ siècle révèle une préférence nettement marquée pour le continent européen (près de $80 \%$ des sociétés exercent leurs activités dans des pays d'Europe ${ }^{6}$ ). Cette "frilosité" des milieux d'affaires belges à investir dans les pays d'outre-mer s'explique, entre autres, par le risque plus grand que comporte un investissement dans les pays lointains et par la difficulté de trouver d'abondants capitaux à risque sur le marché. C'est cette image que le Mouvement Géographique veut modifier, celle d'une Belgique indifférente aux affaires coloniales.

11 Il est donc certain que la naissance de la revue s'inscrit dans le cadre de la politique expansionniste inaugurée par Léopold II au Congo et du mouvement de renaissance géographique qui résulte de la volonté d'élargir le champ de la connaissance au monde 
entier. Mais c'est bien plus encore, l'indifférence de l'opinion publique tout comme celle des milieux d'affaires belges pour les aventures outre-mer qui définissent et justifient l'action, la raison d'être du Mouvement Géographique.

\section{Alphonse-Jules Wauters, maître d'oeuvre du Mouvement Géographique}

12 Alphonse-Jules Wauters, maître d'oeuvre incontestable du Mouvement Géographique naît à Bruxelles, le 13 juin 1845. Issu d'une famille passionnée par l'art et la littérature, A.-J. Wauters y prend rapidement goût lui aussi. On le voit prendre part aux soirées de la Philharmonie dirigée par son père et attiré par les jeux de la scène, s'atteler à l'écriture de pièces théâtrales. Il renonce cependant à la littérature pour se lancer dans une carrière commerciale qui ne sera toutefois que de courte durée en raison de graves problèmes de santé. C'est durant sa longue convalescence qu'il se découvre une passion pour la géographie et les récits de voyage, passion qu'il entretiendra toute sa vie. Comme l'on parle beaucoup du Congo à cette époque, A.-J. Wauters, suit les événements, les étudie avec curiosité et s'entoure de nombreuses cartes de géographie. A la suite de sa maladie, ses affaires avaient périclité et il dut tout liquider. C'est alors qu'il accompagna son frère Emile Wauters lors d'un voyage en Egypte. Ce dernier était peintre et désirait se rendre au Caire pour y trouver l'inspiration d'un nouveau tableau.

A son retour, il accepte la place de critique artistique que son beau-père, M. De Koninck, directeur de l'Echo du Parlement, lui propose. Passionné par cette matière depuis toujours et ayant acquis au contact de son frère, une bonne connaissance dans le domaine de l'art, ses articles sont vite remarqués. Cette nouvelle fonction lui permet de faire la connaissance du directeur de la maison Quantin, importante maison d'éditions artistiques, pour laquelle il rédigera une grande étude sur "L'Histoire de la peinture flamande".

14 Toutefois, sa vie professionnelle prend, une nouvelle fois, un autre tournant puisque c'est à cette époque qu'il participe à la création du Mouvement Géographique, avec les éditeurs Merzbach et Falk, représentants de l'Institut National de Géographie. Tout d'abord rédacteur en chef, Wauters en devient ensuite aussi le directeur sans doute à l'initiative d'Albert Thys lorsque le Mouvement Géographique est repris par la Compagnie du Congo pour le Commerce et l'Industrie. Cette revue, à laquelle il consacra une grande partie de sa vie, reflète sa passion pour la géographie et, plus encore, son enthousiasme à faire connaître la grande oeuvre du Roi au Congo. Selon Lucien Solvay, auteur de sa biographie, les articles de Wauters attirèrent d'ailleurs l'attention de Léopold II, qui le prit très vite en amitié. A cette époque, il semble même que Wauters eut ses entrées à la Cour.

15 Ses articles dont regorgent les colonnes du Mouvement Géographique, ses cartes géographiques soigneusement établies et ses ouvrages concernant le Congo témoignent de l'acharnement dont il fit preuve pour convaincre ses compatriotes de la nécessité d'une colonie pour la Belgique et de l'ardeur qu'il mit à mieux faire connaître le Congo. Toujours dans le même but, on le vit aussi mettre ses qualités d'orateur à contribution lors de nombreuses conférences qu'il donna sur le Congo avec Albert Thys ${ }^{7}$.

Wauters apparaît comme un passionné de géographie. Comme nous le verrons plus loin, son travail, sa persévérance et ses réflexions sur cartes furent couronnées de succès et 
firent de lui, bien qu'il n'y eut jamais mis les pieds, notamment pour des raisons de santé, "le plus grand connaisseur de son temps de la géographie du Congo".

Ses fonctions de journaliste colonial et de géographe devaient sans doute occuper une grande partie de son temps. Mais cela ne l'empêcha pas de se livrer à toute une série d'autres activités. Professeur d'art, auteur d'ouvrages et d'articles sur la peinture flamande, auteur de pièces de théâtre et de contes pour enfants, membre de l'Académie, membre de la Commission directrice des Musées royaux de peinture et de sculpture, de la Société belge de Géographie, il fut aussi secrétaire-général de plusieurs filiales de la Compagnie du Congo pour le Commerce et l'Industrie.

Alphonse-Jules Wauters décède à Ixelles le 25 mars 1916 laissant sa place de rédacteur en chef à Georges Touchard pour les quatre années qui suivirent la fin de la première guerre mondiale avant que le Mouvement Géographique ne disparaisse à son tour.

Le Mouvement Géographique et les nombreux écrits de Wauters relatifs à l'expansion coloniale belge constituent une véritable mine d'informations dans laquelle puisent encore aujourd'hui tous ceux qui s'intéressent à l'histoire de la colonisation belge et à l'histoire de la connaissance géographique du Congo.

\section{Le Mouvement Géographique, une entreprise originale et singulière}

Le Mouvement Géographique s'affirme avant tout comme une revue géographique. Wauters déclare d'ailleurs fièrement que la Belgique est ainsi le premier pays à publier une "gazette géographique". Celle-ci se distingue tout d'abord des bulletins des sociétés de géographie parce qu'elle est un véritable journal mais aussi parce qu'elle poursuit un objectif précis. Si leurs bulletins présentent aussi des comptes rendus de l'activité et des progrès effectués par l'Association Internationale Africaine et ensuite par l'Etat Indépendant du Congo, à côté d'études sur la Belgique et sur d'autres parties du monde, les sociétés de géographie ne feront toutefois pas de l'action coloniale un de leurs objectifs spécifiques. Au contraire, le Mouvement Géographique se fixera comme principal objectif de promouvoir l'expansion coloniale belge en Afrique ainsi qu'en d'autres points du globe.

21 Tout en mêlant connaissances géographiques et promotion de l'expansion coloniale, A.-J. Wauters réunit une abondante documentation. Le Mouvement Géographique se présente ainsi comme une revue spécialisée qui, avec une telle périodicité, ne possède pas d'équivalent dans les autres pays d'Europe à la fin du $19^{\mathrm{e}}$ siècle. Marie-Christine Brugaillère fait d'ailleurs remarquer à ce propos, qu'à cette époque, c'est plutôt une vision exotique et détachée de la réalité que l'on rencontre dans la plupart des journaux s'intéressant à la colonisation'. En outre, Henri Nicolaï cerne parfaitement le caractère original de la publication lorsqu'il affirme qu' “il n'est sans doute pas d'autre exemple d'un journal géographique qui soit si étroitement associé à une entreprise coloniale et qui ait persisté aussi longtemps"10.

Dans le paysage de la presse en Belgique, le Mouvement Géographique apparaît aussi comme une entreprise originale. A l'instar de l'opinion publique, la presse belge est animée des mêmes sentiments d'indifférence et d'hostilité à l'égard de l'oeuvre africaine de Léopold II, souvent jugée néfaste ou irréalisable. Il faut donc attendre l'extrême fin du $19^{\mathrm{e}}$ siècle 
pour voir la grande presse quotidienne consacrer une rubrique spécifique à cette matière qui commence alors à susciter l'intérêt de l'opinion publique.

La presse coloniale belge fait donc son apparition, selon Carl Goebel, précisément avec la création du Mouvement Géographique en $1884^{11}$. Certes l'Association Internationale Africaine a publié périodiquement dès 1878 , les comptes rendus de ses séances. Mais Le Mouvement Géographique est le premier journal qui se consacre presque entièrement aux découvertes géographiques faites par les diverses explorations de cette période et aux activités coloniales de manière générale.

Une autre particularité du Mouvement Géographique est qu'il est resté en activité durant plus de trente ans. Car, si à la fin du $19^{\mathrm{e}}$ siècle et au début du $20^{\mathrm{e}}$ siècle, on vit naître de nombreuses revues se donnant pour objectif de traiter du Congo, la plupart furent éphémères. Elles se créaient et disparaissaient en fonction de l'évolution des événements précis autour desquels elles avaient défini leur action.

\section{L'apport aux géographes et au grand public}

Dès le premier numéro (figure 1), le Mouvement Géographique se présente comme un organe de "vulgarisation" mais aussi de "propagande" et expose clairement son programme. "Journal d'information, il mettra ses lecteurs au courant des dernières nouvelles de tous les points du globe, il les entretiendra des grandes découvertes et des grands travaux d'utilité publique (...). Il désire donc servir de "mémorandum pour l'homme d'étude, le professeur, l'officier, le touriste et renseigner l'homme du monde qui suit en curieux le mouvement géographique, souvent mêlé au mouvement politique (...) Il stimulera l'esprit d'entreprise et soutiendra tous ceux qui s'efforcent d'ouvrir des horizons nouveaux, d'élargir le terrain de notre activité, de pousser le pays à sortir pacifiquement de ses frontières. ${ }^{" 12} \mathrm{~A}$ la lecture des objectifs que le Mouvement Géographique se propose de poursuivre, nous percevons déjà, ce qui deviendra sa principale raison d'être, son leitmotiv durant 35 années: intéresser les Belges à l'entreprise africaine lancée par Léopold II mais plus encore, stimuler l'esprit d'entreprise des milieux d'affaires belges pour les aventures outre-mer. 
Figure 1. Le Mouvement Géographique, 1ère année, $n^{\circ} 1,6$ avril 1884, p. 1.

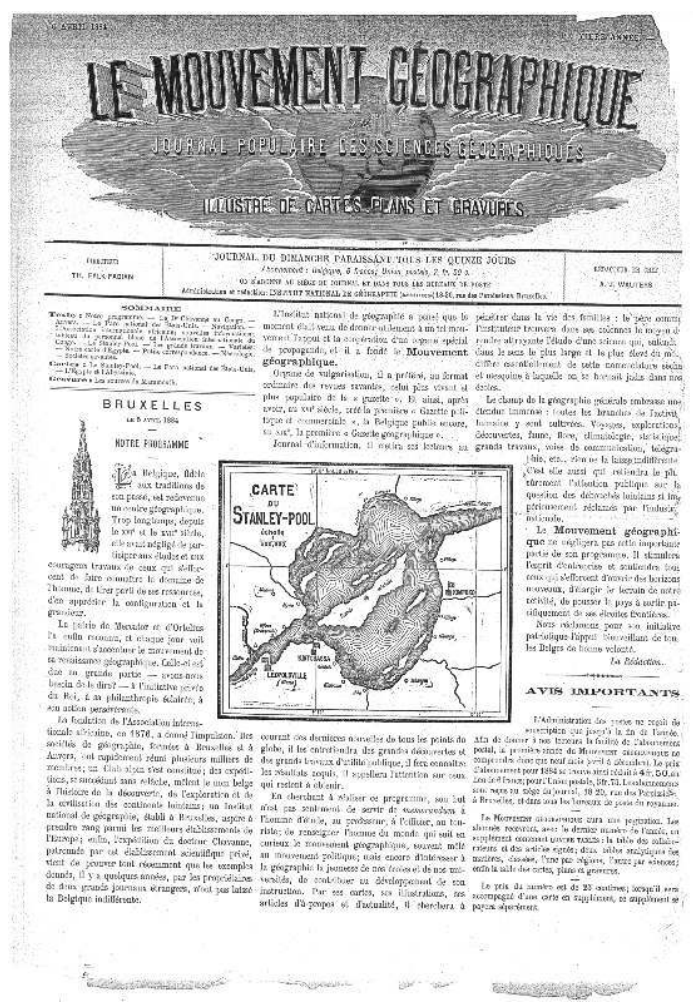

En effet, en considérant la répartition statistique des rubriques par zones géographiques, nous constatons que la part consacrée au continent africain et plus particulièrement à l'EIC fut toujours largement supérieure à celle consacrée au reste du monde comme le montre la figure 2. Celle-ci donne le pourcentage du nombre des articles consacrés aux différentes régions géographiques de 1884 à 1908, date de la reprise du Congo par la Belgique. L'importance attribuée à l'Afrique, et plus encore à l'EIC, est plus forte encore que ce qu'indique le graphique puisque les articles qui les concernent sont d'un volume généralement plus grand que ceux se rapportant aux autres régions. Elle montre clairement que l'objectif affirmé du Mouvement Géographique qui était de mettre ses lecteurs au courant de "tous les points du globe" n'est pas vraiment respecté. Le Mouvement Géographique axe son propos, et ce, dès le départ, sur l'étude d'une région particulière : l'Etat Indépendant du Congo. D'autres régions du monde feront de temps à autre leur apparition sur l'avant de la scène mais uniquement lorsqu'elles intéresseront de près ou de loin la Belgique et les milieux d'affaires belges. La Chine, par exemple, attirera l'attention de Wauters tout le temps que les milieux financiers et industriels belges pourront y voir se développer leurs intérêts avec la construction du chemin de fer reliant Pékin à Hankow. 
Figure 2. Proportion du nombre de rubriques par zone géographique de 1884 à 1908.

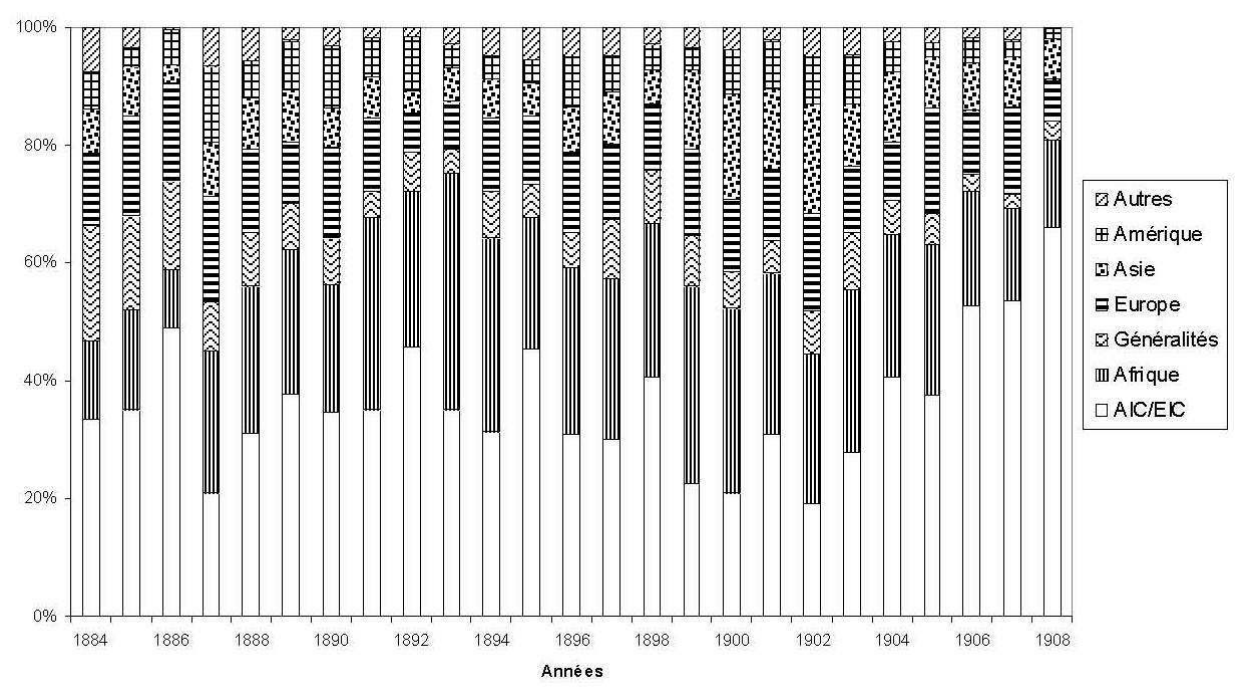

Pour chaque région du monde cependant, A.-J. Wauters rend compte des explorations en cours et des découvertes géographiques. Il procède à une description physique des territoires et de leurs fleuves et donne des informations sur la population, la faune et la flore. Il s'intéresse aux colonies existantes ou en formation et fait état de leurs richesses naturelles, de leurs voies de communication et de ce qu'elles sont susceptibles de rapporter sur le plan économique. Constamment à l'affût d'une situation profitable pour son pays, il porte ainsi son regard sur de nombreuses régions. Ses longues descriptions précises et détaillées dans tous les domaines de la géographie humaine et physique confèrent un cachet scientifique à la revue. Le Mouvement Géographique apparaît ainsi comme une véritable source d'informations. Pourront aller y puiser les passionnés de la géographie et des découvertes géographiques, tout comme les férus de l'aventure coloniale. Toutefois, si le Mouvement Géographique dit vouloir vulgariser les connaissances géographiques et s'adresser à un large public, le contenu et la forme de ses articles en firent surtout une revue destinée à un public averti et bien précis.

Durant trente ans, Wauters récolte tout ce qui s'écrit sur l'Afrique noire et plus particulièrement sur le Congo et rédige d'innombrables articles. Il porte sur des cartes les itinéraires des explorateurs et suit ceux-ci pas à pas dans leurs découvertes. Il leur donne souvent la parole dans le journal. Il met ainsi régulièrement à jour une carte d'Afrique centrale qu'il fournit chaque année en supplément à ses lecteurs. Ses dons de géographe eurent raison d'un des derniers grands "blancs" de la carte d'Afrique, le cours inférieur de l'Uele (figure 3). L'explorateur Schweinfurth croyait que cette rivière rejoignait le lac Tchad. Mais suite au voyage de Grenfell qui avait remonté l'Ubangi jusqu'à ses premiers rapides, Wauters émit l'idée que cette rivière était le cours inférieur de l'Uele. Il argumenta cette thèse en détail dans un article du Mouvement Géographique de 1885. Il faudra pourtant attendre 1887 pour que le voyage de Van Gèle la vérifiât car nombre d'explorateurs étaient restés sceptiques. 
Figure 3. Le Mouvement Géographique, 2ème année, $n^{\circ} 10,17$ mai 1885, p. 1-2.

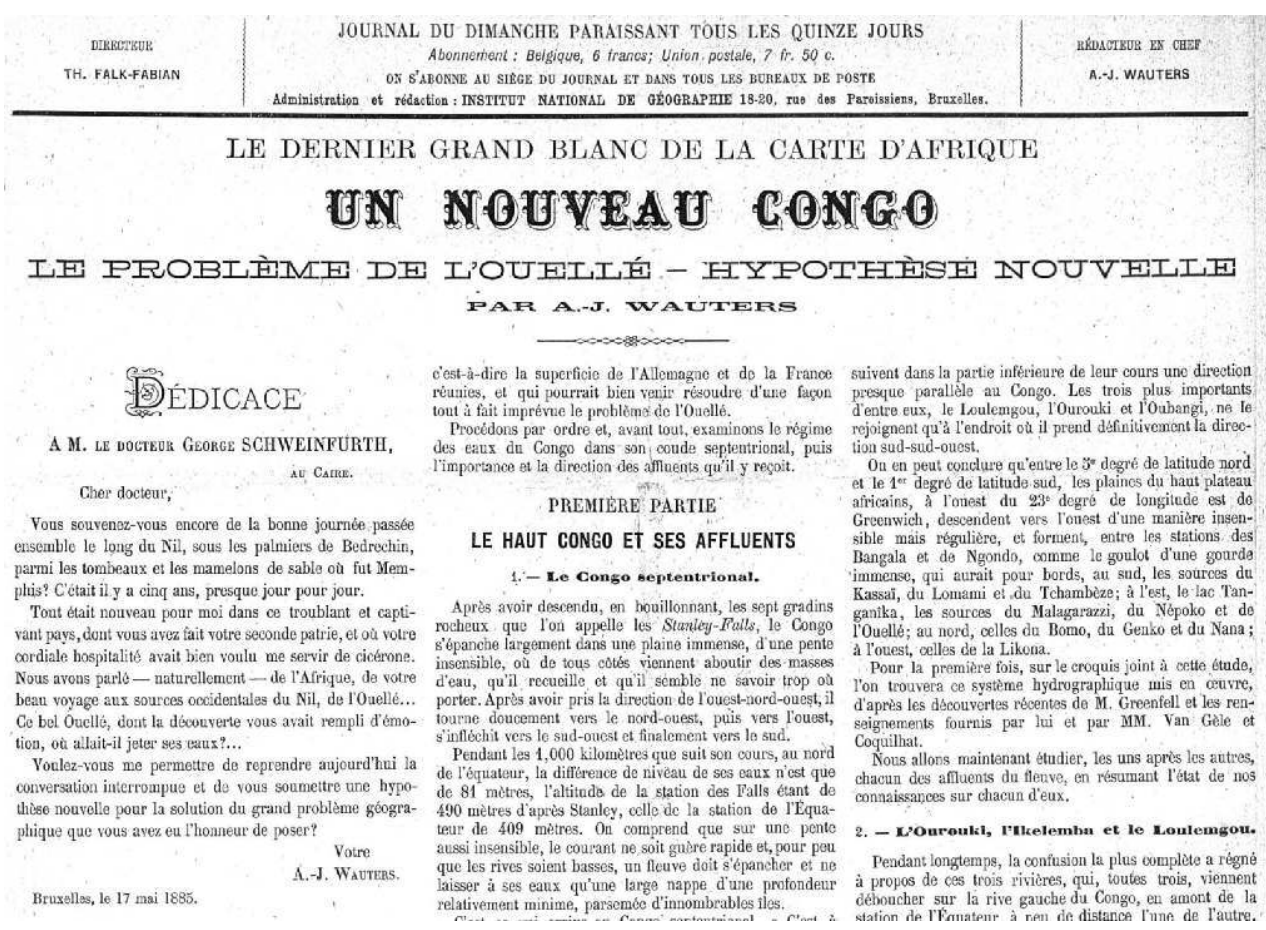

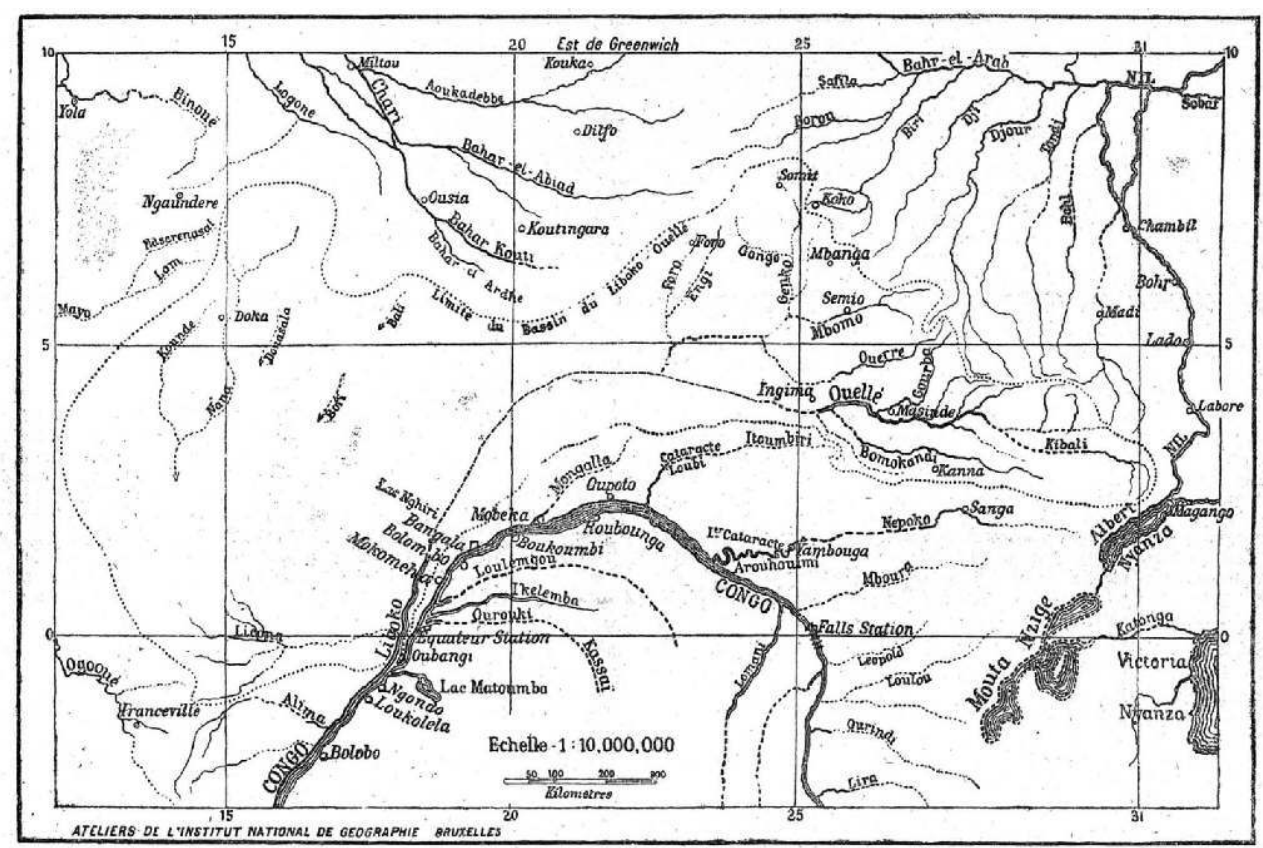

Nouveau tracé hydrographique du Congo septontrional et de ses affuents, dressè d'apres lexploration do M. Greenfell et l'hypothese de M. A.J. Wauters.

(La rivière Liboko eat désignee par les indigenes sous le nom de : riviere a' Oubangi.)

Alors que pour toutes les autres régions du monde, le principal centre d'intérêt est constitué par la politique des grands travaux, lorsqu'il s'agit de l'Etat Indépendant du Congo, le contenu des informations se diversifie en thématiques spécifiques. Ces thématiques témoignent du cours des événements puisque certaines d'entre elles naissent et disparaissent au gré des événements qui les suscitent. De 1884 à 1904, une grande attention est par exemple accordée aux rubriques relatives à la reconnaissance, à la prise de possession et enfin, à la mise en exploitation du Congo. Dans les premières années, qui correspondent à la phase de pénétration, le Mouvement Géographique 
concentre son attention sur les explorations qui sont rapportées en détail à la fois dans les colonnes du journal mais aussi sur les cartes. Ensuite, d'autres sujets viendront prendre la relève, laissant à leur tour la place aux nouvelles informations, aux progrès dans divers domaines et ce en fonction de l'importance que leur accordera le rédacteur en chef du journal. Nous verrons par exemple apparaître une rubrique spécifique consacrée au chemin de fer des Cataractes, de 1887 à 1898, le temps de sa construction; ou encore des articles consacrés au conflit qui éclata entre l'Etat Indépendant du Congo et les sociétés commerciales au sujet de la nouvelle orientation donnée par Léopold II à la politique économique faisant fi de sa promesse de garantir la liberté commerciale au Congo et lésant ainsi grandement les intérêts des sociétés commerciales. Les tentatives de reprise du Congo par la Belgique firent aussi l'objet d'une attention particulière, le Mouvement Géographique publiant un compte-rendu des discussions à chaque négociation que Wauters ne manquait jamais d'assortir de ses commentaires.

Au-delà des passionnés de géographie et des adeptes de la colonisation, le Mouvement Géographique s'adresse clairement à une partie bien précise de l'opinion publique : celle des milieux d'affaires belges. Une rubrique consacrée aux sociétés commerciales fait son apparition dans le journal dès 1886 . Cependant, elle ne prend son véritable envol qu'en 1890 lorsque la revue est reprise par la CCCI et qu'elle représente dès lors explicitement les intérêts des sociétés commerciales. Outre les bilans des diverses filiales de la CCCI, nous y trouvons très régulièrement des informations relatives à l'aspect économique de l'entreprise coloniale. Ces articles concernent les avancées des sociétés commerciales, les réglementations et restrictions que leur impose Léopold II, les richesses dont sont censées regorger de multiples régions, ou encore, les travaux réalisés en matière de voies de communication et ce qu'il reste encore à faire pour favoriser le développement économique du Congo. L'aspect économique et commercial est donc constamment présent dans les colonnes du journal.

\section{La géographie au service de la colonisation}

\section{Le décor et les acteurs : l'Afrique et les pionniers}

31 A l'époque où le Mouvement Géographique fait son apparition, la carte de l'Afrique centrale comporte encore beaucoup de "blancs" et les explorateurs ne sont pas encore venus à bout de tous les mystères du réseau hydrographique ; le bassin du Congo est encore assez mal connu et est loin d'avoir révélé toutes ses richesses. Depuis huit ans déjà, l'Association Internationale Africaine avait été mise sur pied et avait dès sa naissance lancé plusieurs expéditions, relayée ensuite par l'Association Internationale du Congo qui s'était assuré les services de Stanley.

Tel un metteur en scène, Wauters plante d'abord le décor dans lequel se déroulent les explorations et l'entreprise africaine de Léopold II. Le Mouvement Géographique regorge d'articles dans lesquels Wauters décrit inlassablement les régions explorées et transmet à son public sa curiosité, sa fascination pour les nouvelles découvertes et sa soif de connaissances pour ces horizons lointains qui font rêver ses contemporains. Il participe ainsi à l'éveil scientifique dont témoigne le $19^{\mathrm{e}}$ siècle.

Le Mouvement Géographique décrit bien l'atmosphère de découvertes et le foisonnement des explorations des premières années. La moindre nouvelle d'une expédition est mentionnée dans le journal et les rapports des explorateurs sont retranscrits et 
commentés chaque fois sur plusieurs pages. Ces comptes rendus d'expéditions empruntent parfois le style du roman d'aventure, dans lequel le héros, ici l'explorateur, rend compte de chaque péripétie, offre des détails à profusion et fait de son aventure un récit fascinant et parfois effrayant. Wauters loue le courage de ces héros et glorifie leurs actions. Il met souvent Stanley à l'honneur et l'exalte comme le pionnier par excellence de l'entreprise coloniale. Il contribue ainsi à construire le mythe fondateur sur lequel Léopold II bâtira sa grande "oeuvre africaine".

A côté des explorateurs, Wauters n'oublie pas parmi ceux qu'il appelle "les pionniers de l'oeuvre" les hommes politiques et les hommes influents du monde des affaires qui font eux aussi l'objet de nombreuses glorifications. Le journal en fait régulièrement le portrait, et fait état de leurs activités. Wauters, construit ainsi peu à peu de la colonisation "belge" une histoire faite de héros, de gloire et de sentiments élevés.

\section{Le Roi et sa mission philanthropique}

L'un des atouts majeurs de Léopold II dans son action africaine a été la réputation de philanthrope qu'il s'est forgée. Il se présente comme un souverain généreux et désintéressé qui ouvre, à ses frais, l'Afrique centrale à la civilisation et au progrès. Le Roi utilise donc la corde sensible susceptible de faire vibrer l'opinion publique pour sa grande oeuvre "d'humanité" et de "civilisation". A.-J. Wauters a certainement mordu à "l'hameçon" et a soutenu les grandes ambitions humanitaires et scientifiques affichées par le souverain. Dans ses premières années, le Mouvement Géographique apparaît comme un porte-parole, sinon comme un héraut, de la politique africaine du palais. Tous ses articles exaltent "l'oeuvre africaine" du Roi.

Dans les premières années, il est peu question de commerce mais bien de philanthropie. Le discours de Wauters se construit surtout autour de l'idée d'humanisme, de progrès de la civilisation et sur le thème du "devoir" qu'a l'homme blanc d'aller "civiliser" les "races primitives". Le Mouvement Géographique suit cependant avec la plus grande attention toutes les actions de Léopold II sur le plan diplomatique pour faire reconnaitre sa souveraineté sur les territoires qu'il convoite. Déjà, un an avant la création du Mouvement Géographique, Wauters avait réfuté dans un long article de la Société belge de géographie ${ }^{13}$ les prétentions portugaises sur le bassin inférieur du Congo. Au moment de la Conférence de Berlin, il insiste sur les promesses royales de liberté commerciale dans tout le bassin du Congo.

\section{L'énoncé des avantages d'une participation belge à l'action coloniale}

37 A cette époque, comme nous l'avons déjà fait remarquer, les ambitions expansionnistes de Léopold II n'ont que très peu d'écho et ne sont nullement partagées par les milieux d'affaires belges. Le Mouvement Géographique apparait donc comme un outil destiné à combattre cette indifférence. Les aspects économiques de l'expansion vont d'ailleurs peu à peu être mis à l'avant de la scène à la place du caractère philanthropique qui prédominait au début. Tout comme les explorateurs, tels Brazza et Stanley qui désirent convaincre les autorités politiques et les milieux d'affaires de leurs pays en insistant sur les richesses des territoires qu'ils ont découverts, Wauters va développer le mythe des "Indes africaines". La tendance est alors de croire que l'immensité du territoire est gage de richesses inépuisables. Cependant, les difficultés d'accès vers l'intérieur du continent 
restent trop fortes pour que les milieux d'affaires se précipitent sur un marché dont ils évaluent mal les possibilités. L'intérieur du continent reste d'ailleurs très peu connu.

En outre, si Wauters se fait le grand défenseur de la liberté commerciale dans tout le bassin du Congo, dès le départ, il ne cache pas son désir de voir la Belgique y occuper une place privilégiée. Wauters justifie le mouvement d'expansion par la nécessité de trouver de nouveaux débouchés à l'industrie belge. Au moment de la Conférence de Berlin, il insiste déjà sur le caractère belge que doit revêtir l'entreprise: "Aucun des efforts qui sont faits dans le but d'ouvrir à l'industrie des débouchés nouveaux ne peut laisser la Belgique indifférente. Grâce aux capitaux, aux sacrifices et aux dévouements des Belges, l'immense Congo va devenir un marché international. Notre place y est marquée. Quant à venir dire que ce projet est une utopie, c'est une opinion que nous laissons aux infirmes ou à ceux qui, par parti pris, ne veulent pas voir clair." ${ }^{14}$

Nous voyons donc Wauters "marteler" les esprits sur la nécessité pour la Belgique de trouver de nouveaux débouchés et d'engager ses capitaux dans l'entreprise africaine, en réfutant les objections des opposants à l'expansion belge et en accumulant les discours patriotiques. L'idée d'introduire la civilisation en Afrique par le commerce constitue aussi l'un des thèmes clés de la propagande du Mouvement Géographique.

\section{Le financement du Mouvement Géographique}

Comment une telle revue a-t-elle pu subsister pendant une période aussi longue? L'analyse de son discours de propagande nous a tout naturellement conduite à nous interroger sur son financement. Il est en effet très vraisemblable que la publication a été, toujours ou le plus souvent, déficitaire et qu'elle n'a pu se faire sans intervention extérieure. Nous avons donc cherché à savoir qui finançait le Mouvement Géographique, dans quelles conditions et jusqu'à quel point.

\section{Le Mouvement Géographique, publication de l'Institut National de Géographie}

De 1884 à 1889, Le Mouvement Géographique appartient à l'Institut National de Géographie. Cet Institut est une société anonyme fondée le 8 avril 1882 qui a pour objet "l'impression, la publication, l'achat et la vente de tous ouvrages, instruments et appareils se rapportant aux sciences géographiques, cosmographiques, astronomiques et pédagogiques, ainsi que toutes les opérations de fabrication, d'achat et de vente ou autres qui se rattachent directement ou indirectement à ce commerce" ${ }^{15}$. C'est l'Institut National de Géographie qui patronne la mission scientifique menée par le docteur Joseph Chavanne en 1884 et qui publie par exemple la traduction française du livre de Stanley "The Congo and the founding of its Free State" ("Cinq années au Congo. 1879-1884. Voyage. Explorations. Fondation de l'Etat Libre du Congo").

Pour les années antérieures à 1897, nous ne disposons malheureusement d'aucune indication sur la façon dont se faisait le financement du journal ou sur sa santé financière puisque les archives de l'Institut tout comme celles du journal restent à ce jour introuvables. Même lorsque le journal passera sous la direction de la Compagnie du Congo pour le Commerce et l'Industrie, il faudra attendre encore huit années pour le voir apparaître de façon régulière dans la comptabilité de la Compagnie. Nous restons 
toutefois très sceptique quant à la rentabilité du journal lors des treize premières années. En effet, si Marie-Christine Brugaillère voit dans la création, en 1892, d'une seconde revue, le Congo Illustré, le reflet d'un certain succès remporté par le Mouvement Géographique auprès du public ${ }^{16}$, nous doutons par contre du succès financier. D'ailleurs, dès 1895 , un rapport de la CCCI fait état de la mauvaise santé financière du journal et explique le montant élevé de la perte par les "dépenses considérables" occasionnées par les clichés destinés au Congo Illustré. A ce moment, les deux publications ont fusionné. La création du Congo Illustré ne serait donc peut-être pas un signe de succès (du moins sur le plan de la rentabilité) de la première publication mais pourrait tout simplement résulter de la volonté de faire une autre publication, plus attrayante par les illustrations et par les récits plus détaillés d'aventures lointaines fort appréciés à l'époque.

\section{La Compagnie du Congo pour le commerce et l'industrie comme mécène}

En 1890, La Compagnie du Congo pour le Commerce et l'Industrie reprend le Mouvement Géographique à son compte. A partir de 1897, nous disposons d'archives comptables. De cette date à 1914, nous avons constaté que le traitement d'A.-J. Wauters et le coût de l'impression du journal par Weissenbruch constituaient la majeure partie des dépenses. Plus de $50 \%$ des dépenses sont consacrées à ces deux seuls postes, atteignant parfois plus de $70 \%$ et même, comme c'est le cas pour l'année $1897,83,58 \%$. Le paiement effectué à l'imprimerie Weissenbruch constitue généralement le poste le plus élevé, s'élevant à $46,06 \%$ de l'ensemble des dépenses en 1900 pour ensuite diminuer au fil du temps et se situer aux alentours puis en dessous des $30 \%$. Le reste des dépenses comporte les frais de service et d'entretien payés à la Compagnie du Chemin de fer du Congo, le journal occupant sans doute ses locaux; ainsi que les rémunérations de Charles Bulens pour l'impression des cartes et des établissements Jean Malvaux pour la confection des clichés. Parmi les autres dépenses, il faut mentionner l'achat de différents journaux, revues et livres mais cela apparaît tout à fait secondaire.

Les principales sources de revenus sont, quant à elles, en grande partie le produit des abonnements au journal et des annonces. De 1897 à 1900, le produit des abonnements couvre plus de $50 \%$ et même près de $60 \%$ des dépenses. Par contre, à partir de 1901, le produit des abonnements compensera de moins en moins les dépenses. En 1903, il ne les couvre plus qu'à $35,90 \%$ et en 1912 à $23,30 \%$. Les annonces couvrent une part non négligeable des dépenses (entre $8 \%$ et $20 \%$ ). La vente de cartes, de timbres-poste oblitérés et de numéros à la pièce du journal constitue aussi une rentrée d'argent dont le montant reste toutefois très bas.

Suite à cet examen, nous pouvons voir que, tout au long de cette période, le Mouvement Géographique accuse des pertes élevées. Cette constatation est primordiale puisqu'elle pose automatiquement la question de son maintien durant les 35 années de son existence. La CCCI a donc été forcée d'intervenir financièrement pour combler le déficit accusé chaque année par le journal, sans quoi sa publication aurait été interrompue. D'autant plus que le déficit a été particulièrement élevé puisque l'intervention financière de la CCCI s'est élevée en 1897 à 22,74 \% et en 1900 à 14,97 \% et va ensuite ne plus cesser de croitre. Elle devra combler, par exemple, pour l'année 1912, un déficit de 63,14\%.

La situation financière déplorable du journal ne semble pas s'être améliorée après la première guerre mondiale. Georges Touchard, alors rédacteur en chef, en faisant part aux 
lecteurs, en décembre 1922, de l'arrêt de la publication du Mouvement Géographique, le justifie en partie par les difficultés financières "qu'avait à vaincre le Mouvement Géographique pour subsister et garder son caractère, pour ne pas tomber dans le mercantilisme, pour maintenir son indépendance vis-à-vis des puissants du jour" et qui "se trouvaient aggravées par le fait que la Belgique est un petit pays, ne fournissant aux publications scientifiques qu'un public de lecteurs relativement restreint.(...) Le général Thys fit assurer l'existence matérielle du journal par la CCCI, qu'il dirigeait et dont il était l'âme"17.

47 Nous pouvons donc affirmer avec certitude que, durant la majeure partie de son existence, le Mouvement Géographique accusa constamment des pertes élevées et que la CCCI dut sans cesse intervenir financièrement pour combler le déficit. Dès lors, il est évident que, par sa mainmise financière sur le journal, la CCCI a sans aucun doute exercé une influence sur la manière dont les événements et l'information étaient envisagés et relatés.

\section{La question du financement avant 1890}

Avant 1890, alors que le journal est la propriété de l'Institut National de Géographie, il est peu vraisemblable que la situation financière ait été vraiment meilleure. Qui intervenait alors pour combler le déficit? L'absence de tout document d'archives concernant l'Institut National de Géographie ne permet pas de répondre nettement à cette question.

\section{Dans l'ombre : le Roi}

La propagande en faveur de l'action coloniale que fait A.-J. Wauters au sein du journal laisse immanquablement supposer l'existence d'un lien entre le Mouvement Géographique et le Roi Léopold II. En effet, durant les premières années du journal, Wauters défend corps et âme l'oeuvre africaine du Roi et s'efforce sans relâche d'en donner une image positive et attrayante, à tel point que le Mouvement Géographique fut souvent qualifié "d'organe officieux de l'EIC". Cependant, Wauters s'en défendit à plusieurs reprises : une première fois dans un article daté de 1890, où il affirme que "Dans ces derniers temps, différents journaux étrangers nous ont fait l'honneur de signaler notre publication comme étant un organe de l'Etat Indépendant du Congo. Considérant certains de nos articles, ils les ont reproduits comme ayant un caractère quasi-officiel. (...). Nous tenons encore une fois à déclarer là que ces suppositions n'ont rien de fondé. Le Mouvement Géographique est une publication indépendante" ${ }^{18}$. Ensuite, dans sa "Bibliographie du Congo 1880-1895", Wauters rappelle à nouveau que "contrairement à une opinion généralement accréditée, surtout à l'étranger, il [le Mouvement Géographique] n'a jamais été ni subsidié, ni inspiré par le gouvernement de l'Etat Indépendant du Congo" ${ }^{19}$. Néanmoins, cette "confusion" peut apparaitre tout à fait normale puisque l'opinion du Mouvement Géographique s'inscrit à ce moment dans la même ligne de pensée que la politique congolaise du roi Léopold II. Ajoutons cependant qu'à l'époque où le Mouvement Géographique se défend d'avoir un lien quelconque avec le Palais, il est devenu la propriété de la CCCI et lorsqu'il réitère son démenti en 1895 , les louanges de Wauters à l'égard du Roi se sont transformées en d'amères considérations sur le conflit qui oppose alors la Compagnie et l'EIC. 

mainmise royale sur le Mouvement Géographique, certains documents mettent en lumière les "traces" d'une influence exercée par le Roi sur le journal. Dès 1882, lorsque l'Institut National de Géographie voit le jour, nous pouvons déjà entrevoir un lien entre le Palais et le journal. En effet, dans les statuts de cette société anonyme, nous voyons apparaître le nom de S. M. Léopold II en qualité d'actionnaire. Le Roi, sans doute soucieux, comme nous l'avons déjà fait remarquer, de placer son entreprise africaine sous le couvert de la géographie, souscrit alors deux actions sur un total de cent dans cette société qui, deux ans plus tard, créera le Mouvement Géographique ${ }^{20}$.

51 Ensuite, dans une lettre adressée au Roi et datée d'octobre 1891, Albert Thys s'excuse auprès du souverain à propos d'un télégramme (qui donne tout simplement des nouvelles de diverses explorations) mal interprété par le Mouvement Géographique et publié dans ses colonnes: "Je regrette que cette publication ait été faite dans le Mouvement Géographique avant que je n'aie pris les ordres du Roi. La nouvelle étant d'ordre politique, la publication ne devait en être faite qu'avec l'assentiment de Votre Majesté. Cette faute ne se renouvellera plus; j'ai donné des ordres formels à ce sujet." ${ }^{21}$ Ces quelques lignes témoignent donc du "droit de regard" qu'avait Léopold II sur les publications du journal, du moins sur certaines d'entre elles. Nous voyons aussi Albert Thys et à travers lui, la CCCI, tenir véritablement les "ficelles" du Mouvement Géographique dans l'ombre de Wauters.

Enfin, une deuxième correspondance révèle une information capitale sur le financement du journal. Le document date de juillet 1892, alors que le conflit entre la CCCI et l'EIC est déjà engagé au sujet de la nouvelle politique économique poursuivie par le Roi au Congo et que Léopold II vient de céder d'énormes concessions à deux nouvelles compagnies, l'ABIR et l'Anversoise. Dans une lettre au Roi, le secrétaire de l'EIC à Bruxelles, le baron Van Eetvelde rend compte d'une entrevue avec M. Mols, le deuxième homme de l'ABIR, durant laquelle ce dernier avait affirmé qu'il n'était pas influencé par les articles de journaux et qu'il s'était assuré le concours de L'Opinion d'Anvers, ce qui coûterait 2.500 francs à l'Etat. Van Eetvelde propose alors au Roi "d'y consacrer le traitement de Wauters" et ajoute qu'il serait nécessaire de "s'attacher quelques organes par la voie d'un subside, par exemple L'Etoile ou Le Soir" et qu'il faudrait à partir de septembre "mener une campagne de propagande dans la presse". Il ajoute : "nous n'aurons jamais de la part des journalistes un concours absolu si nous ne les payons pas. Ce serait de l'argent bien employé dans les circonstances"22.

53 Cette allusion "au traitement de Wauters" révèle donc que le Mouvement Géographique était bel et bien financé par le Souverain. Bien que nous n'ayons trouvé aucune autre trace de ce financement, il semble que celui-ci fut relativement important, puisque l'on parle carrément d'un "traitement" et non pas d'enveloppes versées de temps à autre. A la proposition de Van Eetvelde, le Roi répondit qu'il voulait bien "faire passer le traitement d'A.-J. Wauters à L'Opinion d'Anvers" ${ }^{23}$. Les propos de Van Eetvelde et ensuite du Roi indiquent donc que la participation royale au financement du Mouvement Géographique a été supprimée en juillet 1892. Cette décision n'est nullement étonnante puisque à cette époque, Wauters, en tant que porte-parole des sociétés commerciales, prend position contre la nouvelle politique commerciale du Roi qui vient de réserver à l'Etat (ou à des sociétés concessionnaires) l'exploitation des produits naturels (essentiellement le caoutchouc et l'ivoire) dans les terres considérées comme vacantes. Wauters rédige sur cette question un nombre impressionnant d'articles, à travers lesquels nous pouvons 
ressentir l'amertume et la colère qu'il éprouve par rapport aux décisions royales. Elle montre aussi que les largesses de Léopold II n'avaient pas pour autant "muselé" le Mouvement Géographique puisque, après avoir chanté les louanges du Roi, il n'hésita pas à prendre ses distances et à critiquer ouvertement la politique économique de l'EIC. Toutefois, il faut nuancer cette indépendance du journal en remarquant que sa prise de position très nette en faveur des sociétés commerciales n'est sans doute pas sans rapport avec la source principale de son financement. Lorsque les intérêts du Roi et de la CCCI divergent, et qu'il perd l'appui financier de Léopold II, Wauters s'attache à mieux servir encore la cause de la Compagnie à laquelle il appartient.

Le montant exact du "traitement" que devait percevoir Wauters n'est pas mentionné dans la correspondance de Van Eetvelde. Celui-ci parle de 2.500 francs qui seraient versés à L'Opinion d'Anvers, publication à laquelle "le traitement de Wauters" serait désormais consacré. Cela peut laisser supposer que le traitement était de l'ordre de 2.500 francs mais aucun autre document ne nous permet de confirmer cette hypothèse. Nous ne savons pas non plus si le Roi finança le journal dès sa création ou s'il en prit l'initiative plus tard, lors de la reprise du Mouvement Géographique par la Compagnie en 1890 puisqu'il était assez proche d'Albert Thys, alors à la tête de la CCCI.

Bien que Léopold II ait participé à la fondation de l'Institut National de Géographie, il nous semblerait toutefois excessif de voir dans la création du Mouvement Géographique une réponse à une sollicitation directe du souverain. On remarquera cependant que le journal apparaît au moment où Léopold II engage la campagne diplomatique qui aboutira à la reconnaissance de sa souveraineté sur le Congo, en marge de la Conférence de Berlin. On ne peut parler non plus d'une mainmise du Roi sur le journal. Cependant on ne peut écarter l'idée que la nature du financement du Mouvement Géographique offre un éclairage ambigu sur le discours de propagande de Wauters. Celui-ci cependant niera toujours avec force que sa revue ait été, de quelconque façon, sous la coupe du palais royal. Il prendra toujours grand soin de présenter le Mouvement Géographique comme une "publication indépendante", "personnelle et désintéressée", n'ayant "jamais eu le besoin de réclamer de quiconque aucune espèce d'inspiration ni de subside". Il revendiquera comme "strictement et exclusivement" siennes "les opinions et les théories qui y sont exposées"24

\section{Faut-il mettre en cause la sincérité de Wauters?}

$\mathrm{Au}$ vu des constats que nous venons de tirer de l'analyse du financement du Mouvement Géographique, une question nous vient immanquablement à l'esprit: Wauters était-il uniquement le porte-parole, le défenseur de ses commanditaires ou était-ce un homme sincère, faisant part de ses convictions personnelles en toute bonne foi indépendamment de ceux qui participaient à la survie financière du journal ?

Il exagère certainement son indépendance et son idéalisme. Mais on remarquera que pendant les premières années du Mouvement Géographique, Wauters était véritablement en phase avec les idées de Léopold II et se mit totalement au service de son "oeuvre" coloniale. Il a en effet soutenu avec enthousiasme l'ouverture du territoire congolais à la civilisation par le commerce, le principe de la liberté commerciale et la nécessaire participation des Belges à l'expansion coloniale. On ne peut donc dire que Wauters fut un journaliste aux ordres du Roi ou "acheté" par ce dernier qui, par ailleurs, soudoya 
régulièrement des journalistes pour défendre certains de ses projets ou pour attaquer un adversaire.

Après 1890, nous y avons fait allusion, Wauters se détache d'ailleurs de la politique du Roi. Son journal est devenu "l'organe des intérêts belges au Congo" comme l'indique son nouveau sous-titre, puis "l'organe des intérêts belges dans les pays d'Outre-Mer". Il s'élève alors contre la politique des monopoles engagée par Léopold II au bénéficie des compagnies concessionnaires ou de l'Etat lui-même ce qui lèse grandement les petites entreprises belges déjà installées sur place et que Wauters défend. En 1892, Wauters consacre de nombreux articles à combattre cette politique. Certes, des accommodements seront trouvés entre l'Etat et les compagnies belges mais Wauters n'acceptera pas que Léopold II ait renié sa promesse solennelle de garantir la liberté commerciale dans le bassin du Congo et il ne verra d'ailleurs comme moyen d'effacer ce manquement à la parole donnée que la reprise la plus prompte possible du Congo par la Belgique. Pendant quinze ans, il ne cessera de réclamer celle-ci dans son journal.

Si Wauters défend donc avec tant d'ardeur les intérêts des sociétés commerciales, ce n'est pas seulement parce que la CCCI finance le Mouvement Géographique, mais surtout, et c'est là notre conviction suite à cette étude, parce que cela correspond à la conception personnelle qu'il se fait de la politique économique à mener dans une colonie. Le Mouvement Géographique change de ton à l'égard du Roi lorsque celui-ci oriente sa politique dans une autre direction; Wauters reste, quant à lui, fidèle à son orientation première, de faire du Congo une entreprise profitable aux Belges et donc ouverte à l'initiative privée.

\section{Conclusion}

Il faut savoir se prêter au rêve lorsque le rêve se prête à nous.

Camus

Bien qu'il ait été créé sur l'initiative de l'Institut National de Géographie, tout au long de son histoire, le Mouvement Géographique semble très souvent l'oeuvre d'un seul homme, d'une seule plume, d'un seul combat. On peut certes considérer qu'il a répondu à un souhait au moins implicite du Roi qui devait y voir un moyen nécessaire pour gagner l'assentiment des Belges à ses projets en Afrique. Mais cet objectif s'est trouvé en accord avec la passion de Wauters pour la géographie, avec son enthousiasme à faire partager les progrès de la connaissance géographique et avec son patriotisme qui lui faisait apparaître la colonisation comme un bien indiscutable pour la Belgique. Pour ce dernier volet, il servit en même temps la cause des compagnies commerciales.

61 Sans doute, l'économique se profile-t-il avec plus ou moins de force en filigrane des actions des hommes et sous-tend-il les visées expansionnistes de la fin du $19^{e}$ siècle. Mais les milieux d'affaires belges et les hommes politiques étaient très réticents à se lancer dans les aventures coloniales. L'action de Wauters, dans le cadre du Mouvement Géographique, a été de chercher à les convaincre. La géographie a tenu une place essentielle dans sar rhétorique. Avec les arguments humanitaires du début de l'entreprise, les préoccupations scientifiques représentées surtout par les découvertes géographiques ont répondu au désir de connaissance et à la soif de progrès présents dans toute une partie de la population principalement cette partie de la bourgeoisie faite de petits fonctionnaires, d'officiers subalternes, de rentiers qui devait constituer le public cible du journal. La géographie, conçue comme une découverte scientifique du monde, a 
permis de diffuser une propagande en faveur de l'action coloniale en ouvrant les esprits à de larges horizons et en les faisant participer, au moins par l'imagination, aux recherches, aux expéditions, aux explorations puis aux premières étapes de la mise en valeur, c'est-àdire de l'exploitation. Wauters et le Mouvement Géographique ont contribué ainsi à sinon à faire accepter, ou du moins à rendre plus familière, l'idée que la Belgique pouvait et devait participer à l'expansion coloniale.

\section{BIBLIOGRAPHIE}

Le Mouvement Géographique, sous-titré “Journal populaire des sciences géographiques, illustré de cartes, plans et gravures", 1ère année (1884)-35e année (1922), (avec une interruption de 1914 à 1918).

Le Congo Illustré (voyages et travaux des Belges dans l'Etat Indépendant du Congo), 1 ère année (1891)-4e année (1894).

\section{DOCUMENTS D'ARCHIVES}

Archives Générales du Royaume

Fonds "Van Eetvelde", n² 29, "Correspondance entre le Secrétaire d'Etat Van Eetvelde et S.M.Léopold II", lettres du 26 et du 27 juillet 1892.

Fonds "Finoutremer", livres comptables de la Compagnie du Congo pour le commerce et l'industrie ,1890-1915.

“Fonds Finoutremer”, livres comptables du Mouvement Géographique, 1908-1910.

Archives du Palais Royal

Archives relatives au développement extérieur de la Belgique sous le règne de Léopold II, correspondance "Albert Thys", n 106/114, lettre d'Albert Thys adressé au Roi, 19 octobre 1891.

Archives du Musée Royal de l'Afrique centrale de Tervuren (section histoire de la période coloniale)

Fonds privé “Albert Thys", lettre de A.-J. Wauters adressée à Albert Thys, Bruxelles, 2 avril 1893.

Bibliothèque de l'Université de Gand

"Institut National de Géographie. Société anonyme établie à Bruxelles constituée par Acte passé devant Me De Doncker, notaire à Bruxelles, le 8 avril 1882, publié au moniteur belge du 22 avril 1882. Statuts", (1882) Bruxelles, M. Weissenbruch.

\section{OUVRAGES ET ARTICLES}

BAUMONT M. (1982), L'essor industriel et l'impérialisme colonial (1878-1904), Peuples et civilisations, XVIII, Paris, PUF.

BEVEL M.-L. (1935), “50 années d'activité coloniale au Congo 1885-1935, La presse coloniale belge" in L'Avenir Belge, p. 290.

BRAILLARD P. et DE SERNACLENS P. (1980), L’Impérialisme, Que sais-je ?, Paris, PUF. 
BRUGAILLERE M.-C. (1993), “Un journal au service d'une conquête : Le Mouvement géographique (1884-1908)” in P.HALEN et J.RIESZ (éd.), Images de l'Afrique et du Congo/Zaïre dans les lettres françaises de Belgique et alentours, Actes du colloque inter-universitaire de Louvain-la-Neuve (4-6 février 1993), pp. 20-35, Textyles, Bruxelles.

CAMBIER R. (1951), “Wauters A.-J.” in Biographie coloniale belge, vol. 2, col. 970, Bruxelles.

CHLEPNER B. S. (1930), Le marché financier belge depuis cent ans, Bruxelles, Librairie Falk fils.

COQUERY-VIDROVITCH C. (2003), "Le postulat de la supériorité blanche et de l'infériorité noire" in FERRO M. (dir.), Le livre noir du colonialisme 16e-20esiècles, de l'extermination à la repentance, Paris, Robert Laffont, pp. 646-684.

DUCHESNE A. (1976), "Les leçons de l'expérience de son père ont-elles entraîné Léopold II dans la voie de la colonisation?", in La Conférence de géographie de 1876, Recueil d'Etudes, pp. 257- 301, Bruxelles, ARSOM.

EMERSON B. (1980), Léopold II : Le royaume et l'empire, Gembloux, Duculot.

GOEBEL C. (1949), "La presse coloniale belge et son évolution" in La Revue coloniale belge, 4eannée, $\mathrm{n}^{\circ} 100$, pp. $766-775$.

GUILLAUME P. (1974), Le monde colonial, $19^{e}-20^{e}$ siècles, Collection U, Paris, Armand Colin.

KURGAN G. (1972), Léopold II et les groupes financiers belges en Chine. La politique royale et ses prolongements (1895-1914), Thèse, Bruxelles, ULB.

KURGAN G. (1988), "Les milieux d'affaires belges et d'outre-mer vers 1885" in Le centenaire de l'Etat Indépendant du Congo. Recueil d'Etudes, pp. 461-464, Bruxelles, ARSOM.

MARCHAL J. (1996), L'Etat libre du Congo : paradis perdu. L'histoire du Congo de 1876 à 1900, Belgique, Cd Paula Bellings.

NDIKUMANA J.-B. (1974), Implantation de la CCCI (Compagnie du Congo pour le commerce et l'industrie) au Congo, 1886-1898, mémoire, Louvain-la-Neuve, UCL.

NICOLAI H. (1994), "Les géographes belges et le Congo" in M.BRUNEAU et D.DORY, Géographies des colonisations $\mathrm{XV}^{e}-\mathrm{XX}{ }^{e}$ siècles, pp. 51-65, Géotextes, Paris, L'Harmattan.

NICOLAI H. (1993), "Le Mouvement Géographique, un journal et un géographe au service de la colonisation du Congo" in Mélanges Pierre Salmon, II, Histoire et ethnologie africaines, vol. XLI, n 1-2, pp. 257-277.

NICOLAI H. (1988), “L'image de l'Afrique centrale au moment de la création de l'Etat Indépendant du Congo" in Le centenaire de l'Etat indépendant du Congo. Recueil d'Etudes, pp. 13-39, Bruxelles, ARSOM.

SOLVAY L. (1925), “A.-J. Wauters” in Annuaire de l'Académie royale des Sciences, des Lettres et des Beaux-Arts de Belgique, col. 169 à 173, 91e année, Bruxelles, Lamartin.

ROEYKENS A. (1963), L'initiative africaine de Léopold II et l'opinion publique belge, Classe des Sciences morales et politiques, XXIX-4, Bruxelles ARSOM.

STENGERS J. (1976), “Léopold II entre l'Extrême-Orient et l'Afrique, (1875-1876)" in La Conférence de géographie de 1876. Recueil d'Etudes, pp. 303-373, Bruxelles, ARSOM.

STENGERS J. (1976), “Introduction” in La conférence de géographie de 1876. Recueil d'Etudes, Bruxelles, ARSOM.

STENGERS J. (1989), Congo, mythes et réalités : cent ans d'histoire, Louvain-la-Neuve, Duculot. 
VAN GEERTRUYEN G. (1991), “Démons sans merveilles, peuples sans histoire. Comment l'Occident a perçu les Africains à travers les siècles", in coll. Racisme. Continent obscur. Clichés, stéréotypes, phantasmes à propos des Noirs dans le royaume de Belgique, pp. 19-44, Bruxelles, CEC-Le Noir du Blanc.

WAUTERS A.-J. (1883), “Le Congo et les Portugais. Réponse au Mémorandum de la Société de Géographie de Lisbonne”, Bulletin de la société belge de géographie, pp. 234-278.

WAUTERS A.-J. et BUYL A. (1895), Bibliographie du Congo 1880-1895. Catalogue méthodique de 3800 ouvrages, brochures, notices et cartes relatifs à l'histoire, à la géographie et à la colonisation du Congo, Bruxelles, Administration du Mouvement Géographique.

WILLAME-SPAEY A. (1976), La presse périodique coloniale belge (1880-1914), mémoire, Bruxelles, ULB.

\section{NOTES}

1. Dans le cadre de ce mémoire d'histoire, nous avons analysé l'intégralité de la collection du Mouvement Géographique. Nous avons par ailleurs consulté les archives comptables du journal lorsqu'il est détenu par la Compagnie du Congo pour le Commerce et l'Industrie ainsi que la correspondance échangée entre le roi Léopold II avec Albert Thys et le secrétaire d'Etat Van Eetvelde. Une étude approfondie du journal et l'examen de ces archives nous ont permis de mettre au jour de nouvelles informations pertinentes complétant ainsi les études menées jusqu'à ce jour sur ce sujet.

2. Java fait alors partie des possessions néerlandaises.

3. J. STENGERS (1976), p. VII.

4. Ibidem, p.VIII.

5. H. NICOLAI (1992), pp. 51-64.

6. G. KURGAN (1972), p. 45.

7. Albert Thys (1849-1915) commença sa carrière coloniale comme secrétaire général de l'Association africaine en 1878 et devint rapidement le bras droit de Léopold II dans le Comité d'Etude du Haut-Congo et dans l'Association Internationale du Congo. Il participa activement à l'édification des projets expansionnistes du Roi qui le nomme officier d'ordonnance en 1879. En 1886, Albert Thys créa la Compagnie du Congo pour le commerce et l'industrie avec l'aide de plusieurs financiers et industriels. Dans cette compagnie, il occupa le poste d'administrateurdélégué et se retrouva aussi à la tête de ses six filiales. Par ailleurs, on le vit participer à l'élaboration d'autres sociétés coloniales qui exerçaient leurs activités au Congo ainsi que dans d'autres pays africains et sur le continent américain. Homme d'affaires très actif, il fonda, en 1899, la Banque d'Outremer et prit les rênes de plusieurs sociétés orientales dont, entre autres, la Compagnie internationale d'Orient. Albert Thys fut, par ailleurs, présent au sein de diverses entreprises charbonnières et métallurgiques belges. En 1889, il exerça la fonction de directeur intérimaire du département de l'Intérieur de l'Etat Indépendant du Congo. En 1890, nous le voyons encore participer à la création du Cercle africain et enfin, en 1912, il créa l'Union coloniale belge.

8. H. NICOLAI (1993), p. 262.

9. M.-C. BRUGAILLERE (1993), p. 27.

10. H. NICOLAI (1994), , p. 63.

11. C. GOEBEL (1949), p. 766.

12. Le Mouvement Géographique, ère année, $n^{\circ}$ 1, 6 avril 1884.

13. A.J. WAUTERS (1883).

14. Le Mouvement Géographique, 1ère année, $n^{\circ} 17,16$ novembre 1884. 
15. Institut National de Géographie. Société anonyme établie à Bruxelles constituée par acte passé devant Maître De Doncker, notaire à Bruxelles, le 8 avril 1882, publié au Moniteur belge du 22 avril 1882. Statuts. Bruxelles, M.Weissenbruch. Un exemplaire de ces statuts se trouve à la bibliothèque de l'Université de Gand. Nous remercions M. Jan Vandersmissen de nous en avoir signalé l'existence et communiquer une copie.

16. M.-C. BRUGAILLERE (1993), p. 25.

17. Le Mouvement Géographique, e année, $n^{\circ}$ 53, 31 décembre 1922.

18. Le Mouvement Géographique, e année, $\mathrm{n}^{\circ} 21,7$ septembre 1890.

19. A.-J. WAUTERS et A. BUYL (1895), p. 2.

20. Le capital est constitué de 100 actions de 5000 francs chacune. Le cinquième est représenté par l'apport que fait la société Merzbach et Falk de l'ensemble de "ses publications ayant trait à la géographie, des objets..., pierres, livres spéciaux, cartes en magasins..., contrats en cours et études préparatoires". (article 5 des statuts). Léopold II souscrit pou 2 actions; Les autres souscripteurs sont des banquiers, qui participent aux syndicats d'émissions d'emprunts publics de l'époque (l'un d'eux, Léon Lambert s'occupait de la fortune privée du Roi, selon l'information que nous a communiquée Mme R. Kurgan), des personnalités de la presse, des officiers supérieurs et un certain nombre de parlementaires. Rien, dans les statuts, n'indique que la société a l'intention de publier un journal.

21. Archives du Palais Royal, archives relatives au développement extérieur de la Belgique sous le règne de Léopold II, $n^{\circ}$ 106/114, correspondance "Albert Thys", lettre d'Albert Thys adressée au Roi, 19 octobre 1891.

22. AGR. Fonds "Van Eetvelde", n²9, "Correspondance entre le Secrétaire d'Etat Van Eetvelde et S.M.Léopold II", lettre du 26 juillet 1892.

23. AGR. Fonds "Van Eetvelde", $n^{\circ} 29$, "Correspondance entre le Secrétaire d'Etat Van Eetvelde et S.M.Léopold II", lettre du 27 juillet 1892.

24. Le Mouvement Géographique, e année, $\mathrm{n}^{\circ}$ 21, 7 septembre 1890.

\section{RÉSUMÉS}

A la fin du $19^{\mathrm{e}}$ siècle, l'Institut National de Géographie à Bruxelles publie Le Mouvement Géographique, sous-titré “journal populaire des sciences géographiques”. Durant près de 35 ans, A.-J. Wauters, son principal maître d'oeuvre, rend compte, avec passion, des découvertes géographiques touchant tous les points du globe. Néanmoins, l'analyse du Mouvement Géographique révèle qu'il ne s'agit pas d'une simple revue de géographie. Il est aussi et surtout un organe de propagande coloniale visant à susciter l'intérêt de l'opinion publique et plus encore des milieux d'affaires belges pour le Congo ainsi que pour toute autre acquisition territoriale. Cet article expose comment la géographie sert surtout de toile de fond à la propagande en faveur de la colonisation dans laquelle Léopold II désire engager son pays. Après une analyse sommaire du contenu du journal et de son évolution au cours de son existence, nous nous interrogeons sur les commanditaires du Mouvement Géographique et nous voyons alors apparaître, dans l'ombre de Wauters, la Compagnie du Congo pour le Commerce et l'Industrie, détentrice du journal dès 1890 mais aussi, de manière plus discrète, le roi Léopold II.

In the late $19^{\text {th }}$ century, the National Institute of Geography in Brussels published "Le Mouvement géographique", subtitled "Journal populaire des sciences géographiques". For almost 
35 years, A.J. Wauters, its major instigator, has described with enthusiasm geographical discoveries all around the globe. Nevertheless, a closer look at the journal reveals it was not a plain geographical publication: the "Mouvement géographique" was also, and first of all, an instrument of colonial propaganda aimed at arousing the interest of the Belgian public opinion and business circles in Congo and in any acquisition of territories.

This paper shows how geography served above all as a backdrop for the propaganda for the colonial aims in which Leopold II wanted to commit his country. After briefly analyzing the contents and evolution of the journal, we wonder who was behind the "Mouvement géographique", and we discover, in the shadow of Wauters, the Compagnie du Congo pour le Commerce et l'Industrie, owner of the journal since 1890, but also, more discreetly, King Leopold II.

INDEX

Mots-clés : colonisation, propagande, presse, Mouvement Géographique, Congo

\section{AUTEUR}

\section{ELISE HENRY}

elisehenry9999@yahoo.fr 